\title{
Heat conduction in a one-dimensional gas of elastically colliding particles of unequal masses
}

\author{
Abhishek Dhar ${ }^{\dagger}$ \\ Raman Research Institute, Bangalore 560080, India.
}

(October 30, 2018)

\begin{abstract}
We study the nonequlibrium state of heat conduction in a one-dimensional system of hard point particles of unequal masses interacting through elastic collisions. A BBGKY-type formulation is presented and some exact results are obtained from it. Extensive numerical simulations for the two-mass problem indicate that even for arbitrarily small mass differences, a nontrivial steady state is obtained. This state exhibits local thermal equilibrium and has a temperature profile in accordance with the predictions of kinetic theory. The temperature jumps typically seen in such studies are shown to be finite-size effects. The thermal conductivity appears to have a very slow divergence with system size, different from that seen in most other systems.
\end{abstract}

PACS numbers: 44.10.+i, 05.45.-a, 05.60.-k, 05.70.Ln

Introduction: The problem of finding a onedimensional system of interacting particles, evolving through Newtonian dynamics, in which Fourier's law of heat conduction holds, has attracted considerable interest in recent years [1]. One of the first studies in the field was the work of Rieder et al [2] who obtained the exact nonequilibrium steady state (NESS) of a chain of coupled harmonic oscillators. They found a constant temperature profile in the system and a heat current that was independent of system size. This result was not too surprising, considering that the harmonic chain is integrable and there is no scattering of phonons. Since then, a large number of studies have looked at the effects of introducing impurities, nonlinearity and external potentials $[3-8]$. The specific question asked in most of these studies is the following. Defining the thermal conductivity $K$ of a system through the linear response formula $j=-K(T) \nabla T$, where $j$ is the the current and $T$ the local temperature, is there a one-dimensional model which gives a finite $K$ ? Contrary to initial expectations, it was found that adding impurities and/or nonlinearity into a system did not result in finite $K$. The first model in which a finite $K$ was found was the so-called ding-aling model [4] in which alternate particles on the line are bound to harmonic springs and the other particles move freely between pairs of the bound particles. Numerical studies of several other models have also given finite conductivity [9]. A common feature in these models is the presence of external potentials which lead to momentum nonconservation. Recently it has been proved rigorously [10] that the conductivity as given by the Green-Kubo formula always diverges in one-dimensional momentum conserving systems with finite pressure. Two recent studies [11] have reported finite conductivity in momentumconserving systems but both of these have vanishing pressure and so there is no contradiction.

While the proof of anamalous conductivity in momentum conserving systems has been a significant progress, there remain many issues that still need to be addressed. First of all, the proof uses the Kubo formula and this is not fully satisfactory since the validity of the Kubo formula, even in the limit of arbitrarily small temperature gradients, has never been established rigorously. It may be noted that any derivation of the Kubo formula for thermal conductivity essentially makes the following assumptions: (i) the NESS is in local thermal equilibrium (LTE) (ii) that Fourier's law is valid and (iii) regression of equilibrium fluctuations occur in the same way as nonequilibrium processes 12]. These assumptions are physically motivated but may not be true in all cases. Secondly we note that while the focus of most of the work on heat conduction has been addressed to obtaining Fourier's law, the more general problem is one of understanding the nonequilibrium energy current carrying state of a many-body system. For example one important question is the existence or otherwise of LTE in the steady state. Thus one would like to know if it is possible to define a slowly varying temperature field which determines all other local properties in the system. This point has not attracted much attention, even though it is quite crucial even for stating Fourier's law and using results of linear response theory. Naively one might expect that if thermal currents in the system vanish in the thermodynamic limit, then LTE should hold but this has been shown not to be true always [13]. Another interesting question is the determination of the temperature profile itself. These questions are clearly of interest and can be asked independently of whether or not Fourier's law holds. Finally we note that numerical studies of heat conduction are problematic for several reasons. One needs long time numerical solution of nonlinear differential equations which is time-consuming and not very accurate. This, in addition to long equilibration times typically occuring in such systems, restricts one to small system sizes. Also the treatment of boundary related problems, such as that of temperature jumps, is 
not straightforward. Hence it has often been difficult to arrive at correct conclusions from numerical studies and it is desirable to have more accurate studies.

In this paper we study heat conduction in a system of hard elastic particles of unequal masses moving on a one-dimensional line. The only interaction between the particles is through elastic collisions. This model was first considered by Casati [14] as a possible candidate for obtaining Fourier's law but the numerical results were insufficient to draw any definite conclusions. More recently this model has been studied by Hatano [7] who obtained a diverging conductivity. The equal-mass case with dissipation was studied by Du et al [8] who obtained a rather surprising NESS which implied a breakdown of usual hydrodynamics.

Here we present extensive and accurate numerical work on this model and also an analytic formulation of the BBGKY-type. Our aim has been to give a more detailed characterization of the NESS than has been previosly done. The present model is particularly suitable for this purpose for two reasons: (i) simulations of this model do not require a numerical solution of nonlinear differential equations and it is possible to obtain very accurate results, (ii) analytically the BBGKY hierarchy has a comparatively simpler structure and some exact statements can be made. The most interesting result obtained is that the steady states for the case of equal masses and the case with arbitrarily small mass differences are completely different. In the thermodynamic limit the latter case exhibits LTE and the temperature profile approaches a form predicted by kinetic theory for a one-dimensional gas. This is surprising since kinetic theory predicts a finite conductivity with a $T^{1 / 2}$ dependence on temperature. On the other hand our model is momentum conserving and the proof for diverging Kubo conductivity holds. In our finite size studies we find a slow divergence of the conductivity $\left(\sim L^{\alpha}\right.$ with $\left.\alpha<0.2\right)$. Our work also clarifies some of the problems related to boundary effects. The jumps in temperature at the boundaries are shown to be finite-size effects which are studied systematically.

Definition of model: We consider $N$ point particles numbered $i=1, \ldots N$ moving in a one-dimensional box extending from 0 to $L$. The mass, position and velocity of the $i$ th particle are denoted by $m_{i}, x_{i}$ and $u_{i}$. The only interaction between particles is through elastic collisions. After a collision between particle $i$ and $(i+1)$, the new velocities are obtained from momentum and energy conservation and given by the linear equations:

$$
\begin{aligned}
u_{i}^{\prime} & =\frac{m_{i}-m_{i+1}}{m_{i}+m_{i+1}} u_{i}+\frac{2 m_{i+1}}{m_{i}+m_{i+1}} u_{i+1} \\
u_{i+1}^{\prime} & =\frac{2 m_{i}}{m_{i}+m_{i+1}} u_{i}-\frac{m_{i}-m_{i+1}}{m_{i}+m_{i+1}} u_{i+1} .
\end{aligned}
$$

Between collisions the particles travel with constant velocity. The coupling to heat baths is implemented by using Maxwell boundary conditions. Thus whenever a particle of mass $m$ collides with a wall at temperature $T$, it is reflected back with a velocity chosen from the distribution $P(u)=(m|u| / T) \exp \left(-m u^{2} /(2 T)\right)$. The temperatures at the two ends are taken to be $T_{1}$ and $T_{2}$.

The case when all particles have equal masses can be solved easily and behaves similarly to the ordered harmonic springs case [2]. The "temperature" profile is flat (with the value $\sqrt{T_{1} T_{2}}$ ), current is independent of system size and there is no LTE. The equal-mass problem is integrable and essentially reduces to a single-particle problem and so the results are not surprising. As soon as the masses are made different, the system becomes nonintegrable and is expected to have good ergodicity properties 15], and correspondingly the NESS should be very different.

$B B G K Y$-type equations: In principle, a complete solution of the heat conduction problem could be obtained from the steady-state solution of the master equation for evolution of the $N$-point distribution equation $\rho\left(\left\{x_{l}, u_{l}\right\}, t\right)$. In practice this is difficult and a simpler approach is to work with the so-called BBGKY hierarchy which deals with reduced distribution functions [16]. Let us first make the following definitions: $\rho_{l}(x, u, t)=\left\langle\delta\left(x-x_{l}\right) \delta\left(u-u_{l}\right)\right\rangle, \rho_{l, l+1}\left(x_{1}, u_{1} ; x_{2}, u_{2}\right)=$ $\left\langle\delta\left(x_{1}-x_{l}\right) \delta\left(u_{1}-u_{l}\right) \delta\left(x_{2}-x_{l+1}\right) \delta\left(u_{2}-u_{l+1}\right)\right\rangle$, where $\langle A\rangle=\int \rho\left(\left\{x_{l}, u_{l}\right\}, t\right) A\left(\left\{x_{l}, u_{l}\right\}\right) \prod_{l} d x_{l} d u_{l}$. Further we define $p_{l, l+1}\left(x, u_{1}, u_{2}\right)$ as the number of collisions per unit time occuring at $x$ between the $l$ th and $(l+1)$ th particles with respective velocities $u_{1}$ and $u_{2}$. Clearly $p_{l, l+1}\left(x, u_{1}, u_{2}\right)=\rho_{l, l+1}\left(x, u_{1} ; x, u_{2}\right)\left(u_{1}-u_{2}\right) \theta\left(u_{1}-u_{2}\right)$. In terms of these the BBGKY-type equations relating one-point functions to two-point functions are the following:

$$
\begin{aligned}
& \partial \rho_{l}(x, u, t) / \partial t+u \partial \rho_{l}(x, u, t) / \partial x= \\
& \int p_{l-1, l}\left(x, u_{1}, u_{2}\right) \delta\left(u_{2}^{\prime}-u\right) d u_{1} d u_{2}-\int p_{l-1, l}\left(x, u_{1}, u\right) d u_{1}+ \\
& \int p_{l, l+1}\left(x, u_{1}, u_{2}\right) \delta\left(u_{1}^{\prime}-u\right) d u_{1} d u_{2}-\int p_{l, l+1}\left(x, u, u_{2}\right) d u_{2} .
\end{aligned}
$$

These equations hold for $x \neq 0, L$. At the boundaries, the distribution functions satisfy appropriate boundary conditions.

The physical observables that we will be interested in are the particle density $n(x, t)$, the energy density $\epsilon(x, t)$ and the energy current density $j(x, t)$. These can be expressed in terms of the one-point functions $\rho_{l}(x, u, t)$. Thus we have:

$$
\begin{aligned}
& n(x, t)=\left\langle\sum_{l} \delta\left(x-x_{l}\right)\right\rangle=\sum_{l} \int \rho_{l}(x, u, t) d u \\
& \epsilon(x, t)=\left\langle\sum_{l} \frac{m_{l} u_{l}^{2}}{2} \delta\left(x-x_{l}\right)\right\rangle=\sum_{l} \frac{m_{l}}{2} \int u^{2} \rho_{l}(x, u, t) d u \\
& j(x, t)=\left\langle\sum_{l} \frac{m_{l} u_{l}^{3}}{2} \delta\left(x-x_{l}\right)\right\rangle=\sum_{l} \frac{m_{l}}{2} \int u^{3} \rho_{l}(x, u, t) d u
\end{aligned}
$$


The temperature field $T(x)$ is defined as $T(x, t)=$ $2 \epsilon(x, t) / n(x, t)$. Our first result is the following exact current conservation equation: $\partial \epsilon(x, t) / \partial t+\partial j(x, t) / \partial x=0$. This result is easily obtained by multiplying Eq. (2) by $m_{l} u^{2} / 2$, integrating over $u$, and summing over all l. There is a pairwise cancellation of all terms on the right-hand side. Similarly by multiplying Eq. (2) by $m_{l} u / 2$, integrating and summing gives, in the steady state: $\partial \epsilon(x) / \partial x=0$. Thus for any choice of masses $\left\{m_{l}\right\}$, the energy density in the steady state is constant in space. Physically the constancy of energy density follows from the constancy of pressure and the linear relation between the two quantites in an ideal gas. However this does not imply a constant temperature profile since the temperature also depends on the number density $n(x)$ which is not constant.

From the fact that the dynamics [Eq. (11)] is invariant under a constant scaling of the masses, it follows that the temperature profile does not change under $m_{i} \rightarrow \nu m_{i}$. Also from the boundary conditions it is easily shown that $T\left(\nu T_{1}, \nu T_{2}, x\right)=\nu T\left(T_{1}, T_{2}, x\right)$. From now on, we shall consider the dimer case where we consider only particles of two different masses $m_{1}$ and $m_{2}$ placed alternately on the line. Because of the above scaling properties the only independent variables are the ratios, $m_{2} / m_{1}$ and $T_{2} / T_{1}$, and $N$. We will henceforth consider the case $m_{1}=1$, $m_{2}=m, T_{1}=2$ and $T_{2}=8$.

Numerical results: In our numerical simulations we let the system evolve with the appropriate boundary conditions and compute time-averages of various quantities in the steady state. The time evolution does not require numerical solution of differential equations since the exact solution is essentially known. The system is evolved by computing successive collision times and updating velocities using the collision rules Eq. (11). The only errors are those due to round-off. We have verified that the simulations reproduce all the exact known results both for the equal and the unequal mass cases.

In our simulations we vary $\delta=m-1$ and $N$. The mass values $\delta=0.078,0.11,0.22,0.44$ were studied for particle numbers $N=41,81,161,321,641$ and 1281 . The size of the boxes were changed so that in all cases the average density of particles was fixed at 2 . The number of particles is chosen to be odd so that at both ends the particles in contact with the bath have the same mass. The number of collisions over which the averaging is done was between $10^{9}-10^{10}$. In all cases we checked that increasing the time of averaging by a factor of 10 did not significantly change the data.

In Fig. (11), we plot the steady state temperature profiles at different system sizes for $\delta=0.22$. The temperature has a smooth and continuously varying profile. There is a jump at the boundaries which decreases as the system size is increased and is expected to vanish in the thermodynamic limit when the current also becomes vanishingly small. We find that this is true for any non-zero $\delta$. For smaller $\delta$ one needs to go to larger system sizes to get the same temperature profile. Infact for small $\delta$ and large $N$ the temperature-profile depends on $\delta$ and $N$ only through the scaling combination $\delta^{2} N$. This is illustrated in Fig. (2) where we plot data corresponding to five different values of $\delta$, each with a different $N$, chosen such that $\delta^{2} N$ is the same. Note that for $\delta=0$, the temperature profile (which is flat and given by $T=\sqrt{T_{1} T_{2}}$ ) and the energy current are both independent of system size; thus $\delta=0$ is a singular point, while the steady state in the limit $\delta \rightarrow 0, N \rightarrow \infty$ with $\delta^{2} N$ constant is quite different and nontrivial.

For fixed value of $\delta$, as we increase $N$ the temperature profile approaches a limiting form. Quite amazingly this limiting form seems to be exactly one that would be predicted by kinetic theory. We recall that kinetic theory for a one-dimensional gas predicts Fourier behaviour with $K \sim T^{1 / 2}$ and hence a nonlinear temperature profile $T_{k}(x)=\left[T_{1}^{3 / 2}(1-x / L)+T_{2}^{3 / 2} x / L\right]^{2 / 3}$. This has been plotted in Fig. (11). We find that the following scaling form gives a reasonable collapse of our data:

$$
T(x, N, \delta)=T_{k}(x)+\frac{1}{\left(\delta^{2} N\right)^{\gamma}} g(x) .
$$

The inset in Fig. (11) shows the collapse of data for $\delta=$ 0.22 obtained using the above scaling form with $\gamma=0.67$.

We now look at the dependence of $K$ on system size. In Fig. (3) we plot $j$ versus $L$ for $\delta=0.22$. It is clear from the data that the conductivity which is proportional to $L j$ has a slow divergence given by $K \sim L^{\alpha}$ with $\alpha<0.2$. We note that this is significantly different from the system size dependence of $K \sim L^{0.4}$ found by Hatano in the same model [7] and also in other momentum-conserving systems like FPU and the diatomic Toda [5, [7].

Finally we have checked for LTE: to do so we compute the steady state expectation $u^{(4)}(x)=\left\langle\sum_{l} m_{l} u_{l}^{4} \delta(x-\right.$ $\left.\left.x_{l}\right)\right\rangle$. If there was LTE, this quantity would be determined by the local temperature $T(x)$. In Fig. (丮) we plot $u^{(4)}(x)$, as determined directly by taking time averages and also the value predicted from the local temperature $T(x)$. We see that at large system sizes LTE is indeed achieved. We also find that for smaller values of $\delta$, one needs to go to larger system sizes to get LTE.

In summary we have studied heat conduction in the unequal mass problem which appears to be the simplest nontrivial deterministic system, in one dimension, for which a very detailed investigation of the NESS can be made. Our study shows that a meaningful hydrodynamic description of the steady state is possible even in a situation where (presumably) $K \rightarrow \infty$ in the thermodynamic limit. It is clear that further studies of this model can throw much light on the difficult problem of transition from the microscopic to macroscopic description in the context of nonequilibrium phenomena.

I thank D. Dhar and M. Rao for many valuable suggestions. I also thank J. Das, C. Dasgupta, R. Pandit, S. 
Ramaswamy and B. S. Shastry for discussions.

$\dagger$ Also at the Poornaprajna Institute, Bangalore. dabhi@rri.res.in

[1] F. Bonetto, J. L. Lebowitz and L. Rey-Bellet, mathph/0002052; E. Atlee Jackson, Rocky Mountain Jn. of Mathematics 8, 127 (1978).

[2] Z. Rieder, J. L. Lebowitz and E. Lieb, J. Math. Phys. 8, 1073 (1967).

[3] R. Rubin and W. Greer, J. Math Phys. 12, 1686 (1971); A. J. O'Connor and J. L. Lebowitz, J. Math. Phys. 15, 692 (1974).

[4] G. Casati, J. Ford, F. Vivaldi, and W. M. Visscher, Phys. Rev. Lett. 52, 1861 (1984).

[5] S. Lepri, R. Livi, and A. Politi, Phys. Rev. Lett. 78, 1896 (1997).

[6] E. A. Jackson and A. D. Mistriotis, J. Phys. Condens. Matter 1, 1223 (1989)

[7] T. Hatano, Phys. Rev. E 59, R1 (1999).

[8] Y. Du, H. Li and L. P. Kadanoff, Phys. Rev. Lett. 74, 1268 (1995).

[9] T. Prosen and M. Robnik, J. Phys. A 25, 3449 (1992); B. Hu, B. Li, and H. Zhao, Phys. Rev. E 57, 2992 (1998).

[10] T. Prosen and D. K. Campbell, Phys. Rev. Lett. 84, 2857, (2000).

[11] C. Giardina et al, Phys. Rev. Lett. 84, 2144 (2000); O. V. Gendelman and A. V. Savin, Phys Rev. Lett. 84, 2381 (2000).

[12] Hydrodynamic Fluctuations, Broken Symmetry, and Correlation Functions, D. Forster (W. A. Benjamin Inc., Reading, MA, 1975).

[13] A. Dhar and D. Dhar, Phys. Rev. Lett. 82, 480 (1999).

[14] G. Casati, Foundations of Phys. 16, 51 (1986).

[15] G. J. Ackland, Phys. Rev E 47, 3268 (1993); R. Artuso, G. Casati and I. Guarneri, Phys. Rev. E 55, 6384 (1996).

[16] "Nonequilibrium Thermodynamics and its statistical Foundations" by H. J. Kreuzer, Clarendon Press, Oxford, (1981).

[17] It is quite interesting to note that the $T^{1 / 2}$ dependence of the conductivity, for arbitrary masses, can also be obtained from the Kubo formula by simply scaling out the temperature.

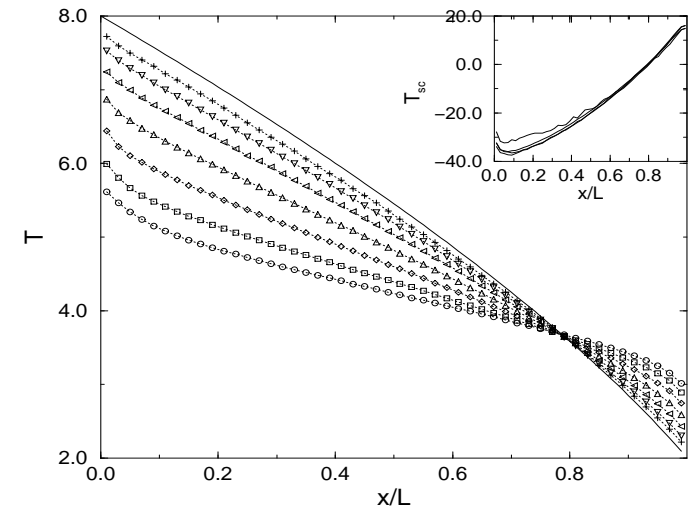

FIG. 1. Temperature profiles for $\delta=0.22$ are plotted for system sizes $N=21$ (indicated by o), 41, 81, 161,321, 641 and $1281(+)$. The solid line is the prediction of kinetic theory. In the inset we have plotted $T_{s c}=N^{0.67}\left(T(x)-T_{k}(x)\right.$ [see Eq. (4)] with the data for $N=161,321,641$ and 1281 .

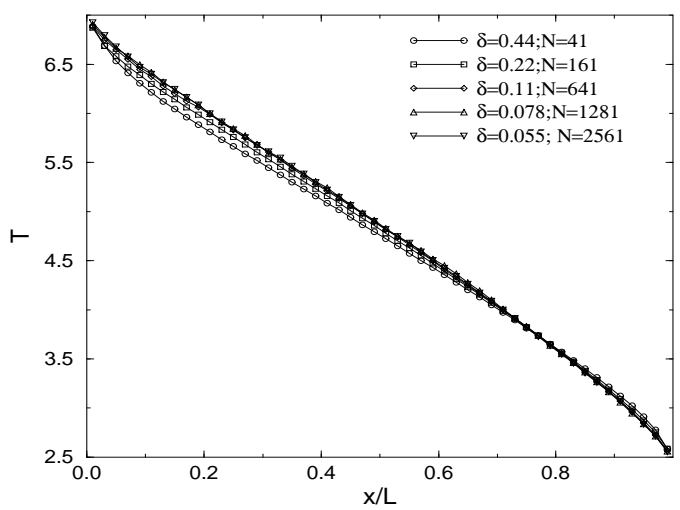

FIG. 2. Temperature profiles obtained with five different sets of values for $\delta$ and $N$ with $\delta^{2} N$ constant. 


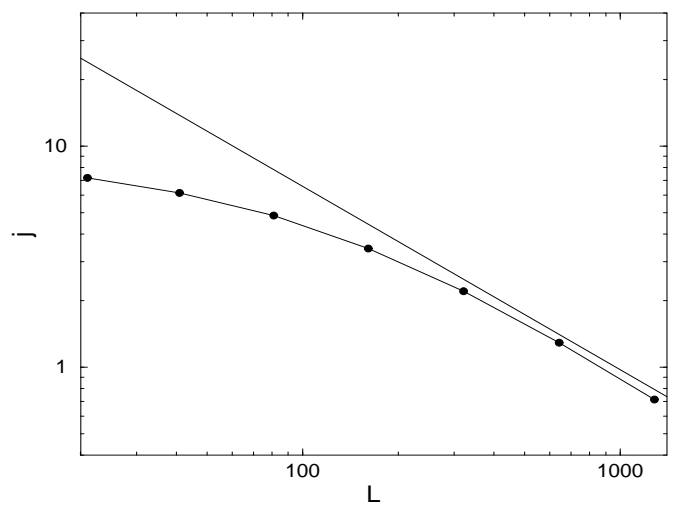

FIG. 3. Plot of $j$ versus $L$ for $\delta=0.22$ The straight line shown corresponds to the decay $j \sim 1 / L^{0.83}$.

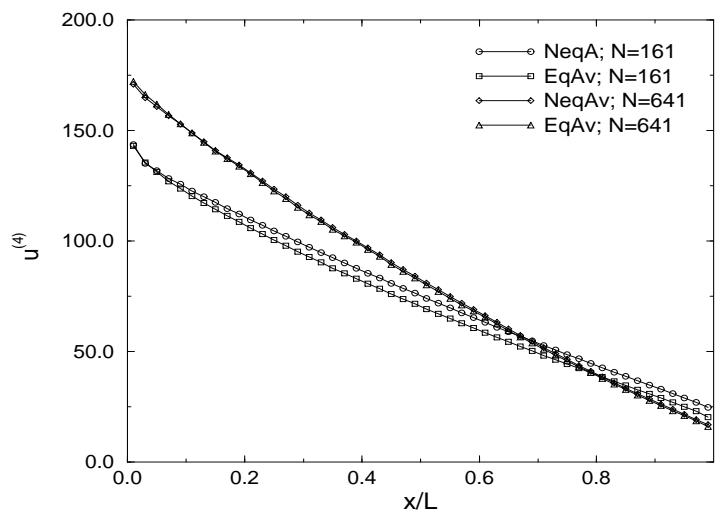

FIG. 4. Plot of $u^{(4)}$ as determined from a direct time averaging (NeqAv) and from the local temperature (EqAv) for two system sizes. For the bigger system, the curves almost coincide. 